\title{
The effect of gender and age on postoperative pain in laparoscopic cholecystectomy: a prospective observational study
}

\author{
Received August 2, 2018 \\ Revised 1st, September 28, 2018 \\ 2nd, October 15, 2018 \\ 3rd, October 18, 2018 \\ Accepted October 19, 2018
}

\author{
Corresponding author \\ Sang Yoong Park, M.D., Ph.D. \\ Department of Anesthesiology \\ and Pain Medicine, Dong-A \\ University College of Medicine, 26 \\ Daeshingongwon-ro, Seo-gu, Busan \\ 49201, Korea \\ Tel: 82-51-240-5390 \\ Fax: 82-51-247-7819 \\ E-mail: Parksy@dau.ac.kr \\ ORCID \\ https://orcid.org/0000-0001-7495-8025
}

\section{Kyoung Lin Chae, Sang Yoong Park, Jeong In Hong, Woo Jae Yim, Seung Cheol Lee, and Chan Jong Chung}

Department of Anesthesiology and Pain Medicine, Dong-A University College of Medicine, Busan, Korea

\begin{abstract}
Background: Gender and age are known factors that affect postoperative pain. The purpose of this study was to investigate the effect of gender and age on postoperative pain and analgesic consumptions after laparoscopic cholecystectomy.

Methods: We studied 240 adult patients (120 female patients and 120 male patients) who underwent elective 3-port laparoscopic cholecystectomy under general anesthesia. The numerical rating scale (NRS) scores were assessed before surgery, when the patient arrived in the recovery room, and at 8,16 , and $24 \mathrm{~h}$ after surgery. Analgesic consumptions in the recovery room and at $24 \mathrm{~h}$ postoperatively were evaluated. We compared the NRS scores and quantity of analgesics between the gender groups and the age groups (20-45, 46-64, and $\geq 65$ years old).

Results: Compared to male patients, female patients had higher NRS scores $(6[5,7]$ vs. $5[4,6] ; P=0.001)$ and required a higher dose of fentanyl $(0.94 \pm 0.47 \mu \mathrm{g} / \mathrm{kg}$ vs. $0.79 \pm 0.41 \mu \mathrm{g} / \mathrm{kg} ; \mathrm{P}=0.011)$ in the recovery room. The younger female patients $(20-45$ and 46-64 years old) required a higher dose of fentanyl than those older than 65 years of age in the recovery room.
\end{abstract}

Conclusions: Female patients exhibit higher NRS scores and greater consumptions of analgesics than male patients immediately postoperatively. Younger female patients require more analgesics than elderly patients in the recovery room.

Keywords: Age; Gender; Laparoscopic cholecystectomy; Postoperative pain.

\section{INTRODUCTION}

Most patients undergoing surgery experience postoperative pain, with $>80 \%$ experiencing moderate to severe pain [1]. Postoperative pain is a major factor causing patient dissatisfaction, delayed recovery, immobility, and prolonged hospital stays; moreover, it is associated with severe complications $[2,3]$. Notable efforts have been made to improve postoperative pain treatment, which include: providing acute pain services, implementing guidelines regarding postop- erative pain, and increasing the use of regional anesthesia techniques; these are currently the most effective efforts for reducing postsurgical pain [4]. Despite these efforts, many patients continue to suffer from severe postoperative pain [2].

Demographic, clinical, and psychological factors have been identified that might be relevant risk factors for the development of severe postoperative pain [5]. The assessment and treatment of postoperative pain requires an individualized approach, as it is difficult to design uniform protocols to sufficiently adapt in accordance with pain sensitivity, which

This is an Open Access article distributed under the terms of the Creative Commons Attribution Non-Commercial License (http://creativecommons.org/licenses/by-nc/4.0) which permits unrestricted non-commercial use, distribution, and reproduction in any medium, provided the original work is properly cited. 
differs among individuals. In a study by Tighe et al. [6], gender, age, and type of surgery were found to affect postoperative pain. Among these factors in previous studies affecting postoperative pain $[7,8]$, female and younger patients showed greater pain intensity. However, Kang et al. [9] showed that male patients reported higher numerical rating scale (NRS) scores than female patients in the recovery room.

Because of different results, there is a need for further studies to evaluate the effects of gender and age on both the intensity of pain and the corresponding analgesic requirements; this might help to modify prescribing habits surrounding analgesic medication. This prospective observational study was undertaken to determine whether there was a difference in the intensity of postoperative pain and analgesic consumptions between female and male patients, and whether the difference is affected by age groups (20-45, 46-64, and $\geq 65$ years old).

\section{MATERIALS AND METHODS}

This prospective observational study was conducted between May 2017 and January 2018. Ethical approval was provided by the institutional review board of our university hospital (no. DAUHIRB-18-066). One hundred twenty female patients and 120 male patients who all underwent elective 3-port laparoscopic cholecystectomy under general anesthesia were enrolled in this study. Preoperatively, all patients received guidance regarding the use of the NRS scores for postoperative pain assessment (from $0=$ no pain to $10=$ the most excruciating pain imaginable).

All patients received premedication with oral diazepam 2-5 mg, $1 \mathrm{~h}$ before surgery. Upon arrival in the operating room, routine monitoring was performed, consisting of electrocardiography, non-invasive blood pressure assessment, and pulse oximetry. When maximal preoxygenation was achieved, induction was performed using intravenous 2-mg/ $\mathrm{kg}$ propofol and $0.9-\mathrm{mg} / \mathrm{kg}$ rocuronium. After induction, i-gel ${ }^{\circledR}$ (Intersurgical Ltd., UK) was used to maintain the airway. Anesthesia was maintained with $40 \%$ oxygen and $1-3 \%$ sevoflurane to maintain hemodynamic variation within $25 \%$ of the preoperative value. At the end of surgery, the residual neuromuscular block was reversed with a mixture of 20-mg pyridostigmine, 0.2-mg glycopyrrolate, and 0.5-mg atropine. All patients were transported to the post-anesthetic recovery unit when they became fully awake. NRS scores were measured by an anesthesia nurse who was not involved in the clinical practice study. If a patient complained of severe pain, an NRS score over 6 , the patient immediately received intravenous $1-\mu \mathrm{g} / \mathrm{kg}$ fentanyl. After patients arrived in the ward, the nurse in charge asked them to report their NRS scores every $8 \mathrm{~h}$ until $24 \mathrm{~h}$ postoperatively. Patients were treated with analgesics in accordance with the routine medication regimen established by the surgeon; if the pain was not se-

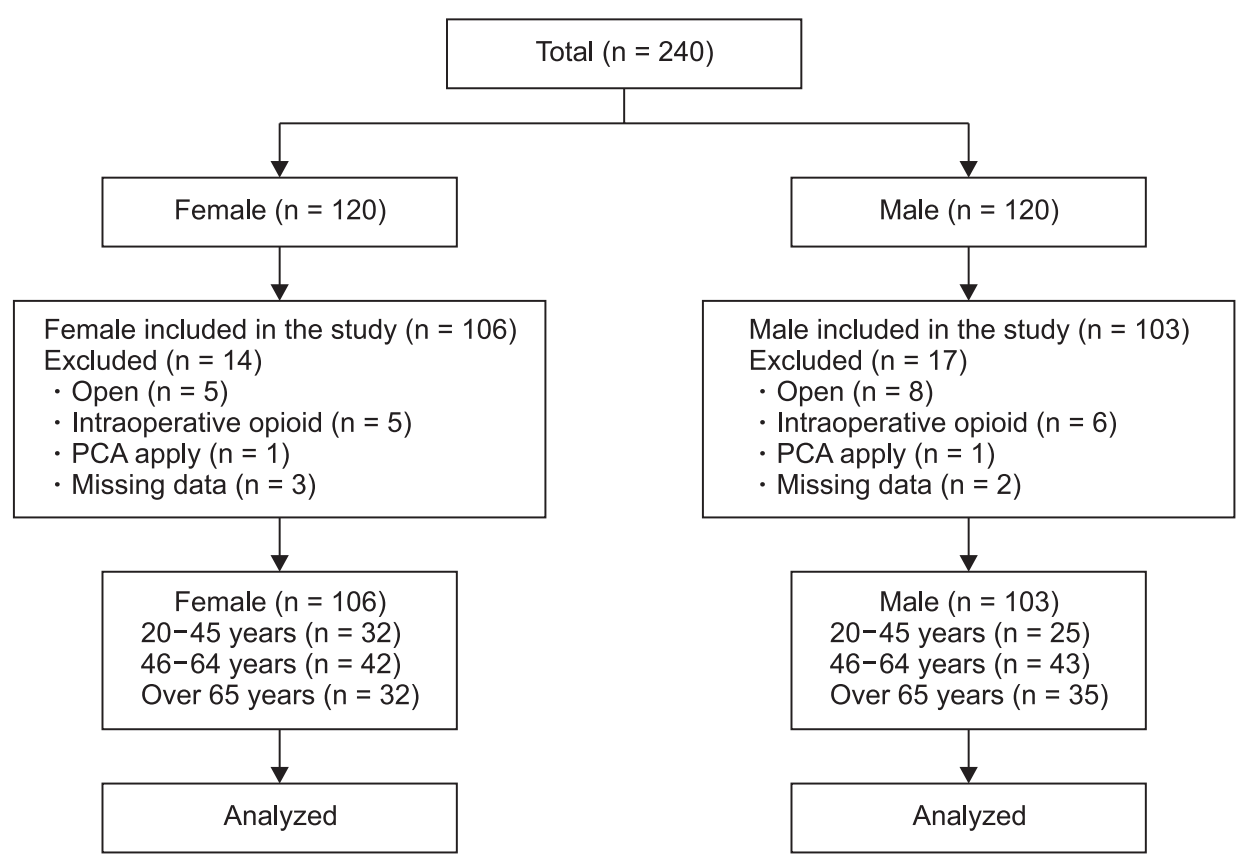

Fig. 1. Patient flow schematic depicting the selection of patients during enrollment and analysis. PCA: patient controlled analgesia. 
vere, patients could refuse medication. Based on the routine medication regimen, patients immediately received 25-mg pethidine intravenously when they were transported to the ward, and then they received 50-mg dexketoprofen every $8 \mathrm{~h}$. Conversely, if the pain was severe, additional analgesics were administered, in accordance with the surgeon's judgment. In the ward, all decisions to administer analgesics were determined by one surgeon who performed the surgery.

The NRS scores and the quantity of analgesics administered were compared according to gender. Further, to determine the degree of pain associated with age, the NRS scores and quantity of analgesics administered were compared among the age groups of $20-45,46-64$, and $\geq 65$ years old, within the same gender.

The sample size was based on data from a pilot study of 20 male patients, wherein we assessed fentanyl consumption $(0.83 \pm 0.45 \mu \mathrm{g} / \mathrm{kg})$. To detect a difference of $10 \%$ in the fentanyl consumption between the groups with a two-tailed $\alpha=$ 0.05 and power of $80 \%$, a sample size of 98 patients would be required. Thus, we recruited 120 patients per group to allow for the potential dropout of subjects.

Continuous data are expressed as mean \pm standard deviation, whereas categorical data are expressed as median (interquartile range). Demographic data and the quantity of analgesics administered between the gender groups were analyzed with the unpaired Student's $t$-test. NRS scores were analyzed by the Mann-Whitney's $U$ test. One-way analysis of variance and Kruskal-Wallis' test with post hoc Bonferroni's method were performed to analyze the quantity of analgesics administered and NRS scores among the age groups, respectively. Data analysis was performed using SPSS software version 23 (IBM Corp., USA).

Table 1. Patient Demographic Data

\begin{tabular}{lccr}
\hline \multicolumn{1}{c}{ Characteristic } & $\begin{array}{c}\text { Female patients } \\
(\mathrm{n}=106)\end{array}$ & $\begin{array}{c}\text { Male patients } \\
(\mathrm{n}=103)\end{array}$ & P value \\
\hline ASA $(1 / 2 / 3)$ & $19 / 64 / 23$ & $9 / 60 / 34$ & 0.056 \\
Age $(\mathrm{yr})$ & $54.6 \pm 16.1$ & $56.7 \pm 15.5$ & 0.321 \\
Weight $(\mathrm{kg})$ & $62.6 \pm 10.3$ & $73.8 \pm 10.8$ & $<0.001$ \\
Height $(\mathrm{m})$ & $1.56 \pm 0.1$ & $1.70 \pm 0.1$ & $<0.001$ \\
Body mass index $\left(\mathrm{kg} / \mathrm{m}^{2}\right)$ & $25.5 \pm 3.7$ & $25.6 \pm 3.1$ & 0.907 \\
Operating time $(\mathrm{min})$ & $40.7 \pm 12.8$ & $44.6 \pm 19.9$ & 0.090 \\
Anesthesia time $(\mathrm{min})$ & $63.8 \pm 14.7$ & $68.3 \pm 19.8$ & 0.068 \\
\hline
\end{tabular}

Values are presented as number or mean \pm SD. ASA: American Society of Anesthesiology physical status classification.

\section{RESULTS}

Among the 240 patients, cases involving conversion laparotomy, treatment with intraoperative opioids because of high blood pressure, postoperative application of patientcontrolled analgesia (patient requirement), and missing data were excluded from this study. Finally, 106 female patients and 103 male patients were included in the study (Fig. 1). Patient demographic data are shown in Table 1. No significant differences were observed for the American Society of Anesthesiologists physical status classification, age, body mass index, operating time, or anesthesia time between female and male patients.

The NRS scores were compared between female and male patients. Fig. 2 shows the differences in NRS scores based on gender: female patients reported higher NRS scores than male patients in the recovery room $(6[5,7]$ vs. $5[4,6], \mathrm{P}=$ $0.001)$. There were no differences between the gender groups in NRS scores preoperatively and at 8,16 , or $24 \mathrm{~h}$ postoperatively.

The quantities of analgesics administered were compared between female and male patients. Female patients received more fentanyl than male patients in the recovery room (0.94 \pm 0.47 vs. $0.79 \pm 0.41 \mu \mathrm{g} / \mathrm{kg}, \mathrm{P}=0.011)$. There were no differences between the groups in the quantities of dexketoprofen and pethidine administered during $24 \mathrm{~h}$ postoperatively.

To compare the effects of age, each gender was divided

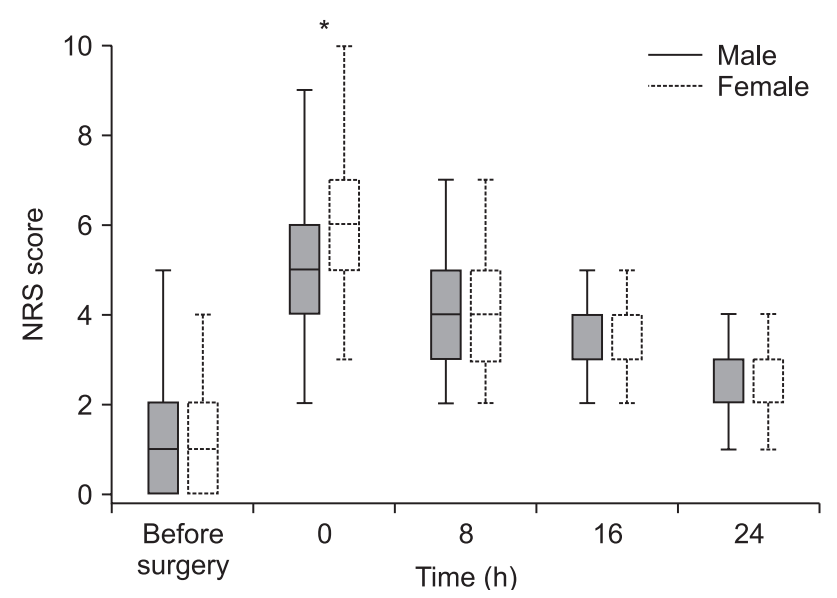

Fig. 2. Graph depicting postoperative numerical rating scale scores at different observation times. Points represent medians. The lower, median, and upper quartiles appear as a box. Minimum and maximum data points are indicated by whiskers. NRS: numerical rating scale. *P $=0.001$ compared to male patients. 
into three groups according to age. Table 2 shows the NRS scores for each age group. There were no significant differences in NRS scores between age groups of the same gender. However, compared to male patients, female patients in the 20-45-year-old group reported higher NRS scores in the recovery room $(6[5,7]$ vs. $5[4,6], \mathrm{P}=0.015)$. Table 3 shows the average quantity of each analgesic administered for each age group. The 20-45- and 46-64-year-old groups received more fentanyl than the $\geq 65$-year-old group among female patients. There was no significant difference in male patients among the age groups. Compared to male patients, female patients in the 20-45- and 46-64-year-old groups received more fentanyl in the recovery room.

\section{DISCUSSION}

In this study, we found a significant difference between female and male patients in postoperative pain and analgesic consumptions in the recovery room. The younger female patients reported a higher dose of fentanyl than elderly patients in the recovery room.

There are two likely reasons for a difference in surgical pain according to gender. First, female patients have a lower pain threshold and lower tolerance for experimental pain than male patients [10]. A meta-analysis of healthy volunteers provided quantitative confirmation of this result. In terms of pain threshold and tolerance, male patients exhibited higher pain threshold than female patients for all types of noxious stimuli, although the largest effect sizes were obtained be-

Table 2. Postoperative Numerical Rating Scale Score by Age Group

\begin{tabular}{|c|c|c|c|c|c|c|c|c|c|c|c|c|}
\hline \multirow[b]{2}{*}{ Variable } & \multicolumn{4}{|c|}{ Female patients } & \multicolumn{4}{|c|}{ Male patients } & \multicolumn{4}{|c|}{ Total } \\
\hline & $\begin{array}{c}20-45 \\
(n=32)\end{array}$ & $\begin{array}{c}46-64 \\
(n=42)\end{array}$ & $\begin{array}{c}\geq 65 \\
(n=32)\end{array}$ & $P$ value & $\begin{array}{c}20-45 \\
(n=25)\end{array}$ & $\begin{array}{c}46-64 \\
(n=43)\end{array}$ & $\begin{array}{c}\geq 65 \\
(n=35)\end{array}$ & $P$ value & $\begin{array}{c}20-45 \\
(n=57)\end{array}$ & $\begin{array}{c}46-64 \\
(n=85)\end{array}$ & $\begin{array}{c}\geq 65 \\
(n=67)\end{array}$ & $P$ value \\
\hline Before surgery & $0(0,2)$ & $1(0,2)$ & $2(0,2)$ & 0.143 & $0(0,2)$ & $0(0,3)$ & $1(0,2)$ & 0.458 & $0(0,2)$ & $1(0,2)$ & $1(0,2)$ & 0.890 \\
\hline After surgery & $6(5,7)^{*}$ & $6(4,7)$ & $5(4,7)$ & 0.482 & $5(4,6)$ & $5(4,6)$ & $5(4,6)$ & 0.920 & $5(4,7)$ & $5(4,7)$ & $5(4,6)$ & 0.568 \\
\hline $8 \mathrm{~h}$ after surgery & $4(4,5)$ & $4(3,5)$ & $4(3,5)$ & 0.448 & $4(3,5)$ & $4(3,4)$ & $4(3,5)$ & 0.452 & $4(3,5)$ & $4(3,5)$ & $4(3,5)$ & 0.367 \\
\hline $16 \mathrm{~h}$ after surgery & $3(3,4)$ & $3(3,4)$ & $3(2,3)$ & 0.405 & $3(3,3)$ & $3(3,4)$ & $3(3,4)$ & 0.180 & $3(3,4)$ & $3(3,4)$ & $3(3,4)$ & 0.899 \\
\hline $24 \mathrm{~h}$ after surgery & $3(2,3)$ & $3(2,3)$ & $3(2,3)$ & 0.898 & $3(2,3)$ & $3(2,3)$ & $3(2,3)$ & 0.350 & $3(2,3)$ & $3(2,3)$ & $3(2,3)$ & 0.438 \\
\hline
\end{tabular}

Values are presented as median $(1 \mathrm{Q}, 3 \mathrm{Q})$. ${ }^{*} \mathrm{P}=0.015$ compared to male patients in the same age group.

Table 3. The Average Quantity of Each Analgesic by Age Group

\begin{tabular}{|c|c|c|c|c|}
\hline Analgesic & Female patients $(n=106)$ & Male patients $(n=103)$ & $P$ value & Total $(n=209)$ \\
\hline \multicolumn{5}{|l|}{ Fentanyl ( $\mu \mathrm{g} / \mathrm{kg})$} \\
\hline $20-45 \mathrm{yr}$ & $1.03 \pm 0.40 *$ & $0.80 \pm 0.41$ & 0.036 & $0.93 \pm 0.42 *$ \\
\hline $46-64 \mathrm{yr}$ & $1.07 \pm 0.46^{*}$ & $0.79 \pm 0.41$ & 0.004 & $0.93 \pm 0.42 *$ \\
\hline$\geq 65 \mathrm{yr}$ & $0.69 \pm 0.47$ & $0.77 \pm 0.43$ & 0.446 & $0.73 \pm 0.45$ \\
\hline$P$ value & 0.001 & 0.962 & & 0.012 \\
\hline Total & $0.94 \pm 0.47$ & $0.79 \pm 0.41$ & 0.011 & $0.87 \pm 0.45$ \\
\hline \multicolumn{5}{|l|}{ Pethidine (mg) } \\
\hline $20-45 \mathrm{yr}$ & $21.09 \pm 9.22$ & $19.00 \pm 14.93$ & 0.518 & $20.18 \pm 11.99$ \\
\hline $46-64 \mathrm{yr}$ & $22.02 \pm 11.31$ & $20.35 \pm 11.25$ & 0.496 & $21.18 \pm 11.25$ \\
\hline$\geq 65 \mathrm{yr}$ & $25.00 \pm 14.20$ & $21.43 \pm 17.30$ & 0.362 & $23.13 \pm 15.89$ \\
\hline Pvalue & 0.377 & 0.814 & & 0.434 \\
\hline Total & $22.64 \pm 11.72$ & $20.39 \pm 14.33$ & 0.214 & $21.53 \pm 13.08$ \\
\hline \multicolumn{5}{|c|}{ Dexketoprofen (mg) } \\
\hline $20-45 \mathrm{yr}$ & $135.94 \pm 51.17$ & $126.00 \pm 57.95$ & 0.495 & $131.58 \pm 53.98$ \\
\hline $46-64 \mathrm{yr}$ & $128.57 \pm 56.46$ & $126.74 \pm 51.58$ & 0.877 & $127.65 \pm 53.73$ \\
\hline$\geq 65 \mathrm{yr}$ & $121.88 \pm 56.71$ & $122.86 \pm 61.05$ & 0.946 & $122.39 \pm 58.57$ \\
\hline$P$ value & 0.594 & 0.953 & & 0.649 \\
\hline Total & $128.77 \pm 54.75$ & $125.24 \pm 55.96$ & 0.645 & $127.03 \pm 55.24$ \\
\hline
\end{tabular}

Values are presented as number or mean $\pm \mathrm{SD}$. $* \mathrm{P}=0.017$ compared to age group ( $>65$ years old). 
tween the sexes for electrical pulses and thermal stimuli [11]. Second, hormonal variation can also lead to gender differences in pain experience. Gonadal hormones are known to modulate pain intensity and to influence sensitivity to opioid analgesics. During the luteal phase of the menstrual cycle, a decreased pain threshold and a corresponding increase in opioid consumption have been reported [12].

Previous studies have shown similar results regarding pain perception differences between female and male patients. Cepeda and Carr [13] evaluated postoperative pain in 423 female patients and 277 male patients in the recovery room. Compared to male patients, female patients reported higher pain scores and required additional morphine for pain relief. Moreover, Zeidan et al. [14] observed that female patients reported higher NRS scores and required greater morphine consumption in the recovery room, following gastric bypass surgery.

In contrast, Theodoraki et al. [15] showed that there was no difference in postoperative pain and morphine consumption between female and male patients undergoing abdominal surgery; however, in contrast to our study, their results were based on relatively older patients. The mean ages of female and male patients in that study were $64.7 \pm 8.9$ and $66.4 \pm 9.0$ years, respectively; these ages were much older than those in the present study $(54.6 \pm 16.1$ and $56.7 \pm 15.5$ years, respectively). Yet, their study was similar to ours in terms of no difference in the quantity of analgesics administered in a comparison between female and male patients in the $\geq 65$-yearold group.

One of the main results of our study was that the older patients reported lower analgesic consumptions in the recovery room. In a study by Tighe et al. [6], older patients reported lower postoperative pain scores, which is similar to the present study's result. Aubrun et al. [12], however, showed a negative correlation between pain scores and age. There are some factors that may contribute to this result, such as psychosocial differences in pain processing, pharmacokinetic or pharmacodynamic changes with age, or the residual effect of hypnotic agents from the surgery [16]. Because of these differences, further investigation will be needed in the future.

On the basis of our results, we decided to change our protocol of postoperative pain management from intravenous fentanyl at a dose of $1 \mu \mathrm{g} / \mathrm{kg}$ to $1.5 \mu \mathrm{g} / \mathrm{kg}$ in female patients younger than 60 years old in the recovery room.
The present study involved certain limitations. First, the sample size was not sufficient to compare according to age groups. In order to reduce biases such as the surgical method, operative time, and anesthetic method, we collected data from the same operation and operator in the same medical institution. The results of this analysis were also based on patient data from one single university hospital with its own standard postoperative pain treatment protocol, which might influence the generalization of our results. Second, use of the NRS scores assumed that pain was a simple condition for patients to assess. Although intensity is a very important aspect of pain, it has been established that pain refers to a varied feeling that cannot be sufficiently categorized under a single descriptor (i.e., intensity) $[17,18]$. This limitation may be minimized through the use of multi-item pain assessment scales, such as the Defense and Veterans Pain Rating Scale [19]. Third, we did not consider the hormonal state of the female patients or the stage of their menstrual cycles; thus, this could be another possible uncontrolled confounding factor for the differences in our results [20]. Fourth, we did not evaluate the effect of oral diazepam as premedication. Because of its antianxiety property and sedative effect, diazepam could affect patients' assessment of their NRS scores. Finally, although there are statistical differences in consumptions of fentanyl administration, it is difficult to say that there were clinically significant differences.

In conclusion, female patients reported higher NRS scores than male patients in the recovery room. It was revealed that gender is an important factor in effective pain management, such that this factor should be considered for its implications in the treatment of pain in female patients. Additionally, there was a significant difference in analgesic consumptions between the female and male patients in the recovery room. Female patients received more fentanyl than male patients in the recovery room in the 20-45- and 46-64-year-old groups. Furthermore, the younger female patients required more analgesics than the elderly patients aged $\geq 65$ years old in the recovery room. The clinical relevance of the statistically significant but only slightly higher consumption of fentanyl in younger female patients than in older patients will require further research. 


\section{ACKNOWLEDGMENTS}

This research was supported by research funds from DonaA University.

\section{CONFLICTS OF INTEREST}

No potential conflict of interest relevant to this article was reported.

\section{ORCID}

Kyoung Lin Chae: https://orcid.org/0000-0001-9132-8279

Jeong In Hong: https://orcid.org/0000-0002-9487-3368

Woo Jae Yim: https://orcid.org/0000-0003-4041-5334

Seung Cheol Lee: https://orcid.org/0000-0001-8669-5517

Chan Jong Chung: https://orcid.org/0000-0002-0236-7135

\section{REFERENCES}

1. Apfelbaum JL, Chen C, Mehta SS, Gan TJ. Postoperative pain experience: results from a national survey suggest postoperative pain continues to be undermanaged. Anesth Analg 2003; 97 : 534-40.

2. Katz J, Seltzer Z. Transition from acute to chronic postsurgical pain: risk factors and protective factors. Expert Rev Neurother 2009; 9: 723-44.

3. Kehlet H, Jensen TS, Woolf CJ. Persistent postsurgical pain: risk factors and prevention. Lancet 2006; 367: 1618-25.

4. Fletcher D, Fermanian C, Mardaye A, Aegerter P; Pain and Regional Anesthesia Committee of the French Anesthesia and Intensive Care Society (SFAR). A patient-based national survey on postoperative pain management in France reveals significant achievements and persistent challenges. Pain 2008; 137: 441-51.

5. Ip HY, Abrishami A, Peng PW, Wong J, Chung F. Predictors of postoperative pain and analgesic consumption: a qualitative systematic review. Anesthesiology 2009; 111: 657-77.

6. Tighe PJ, Le-Wendling LT, Patel A, Zou B, Fillingim RB. Clinically derived early postoperative pain trajectories differ by age, sex, and type of surgery. Pain 2015; 156: 609-17.

7. Zheng H, Schnabel A, Yahiaoui-Doktor M, Meissner W, Van Aken $\mathrm{H}$, Zahn P, et al. Age and preoperative pain are major con- founders for sex differences in postoperative pain outcome: a prospective database analysis. PLoS One 2017; 12: e0178659.

8. Rosseland LA, Stubhaug A. Gender is a confounding factor in pain trials: women report more pain than men after arthroscopic surgery. Pain 2004; 112: 248-53.

9. Kang DH, Kim DS, Kim JD, Kim JW. A comparison of fentanyl and morphine for patient controlled analgesia after laparoscopic cholecystectomy. Anesth Pain Med 2013; 8: 21-5.

10. Fillingim RB, Ness TJ, Glover TL, Campbell CM, Hastie BA, Price DD, et al. Morphine responses and experimental pain: sex differences in side effects and cardiovascular responses but not analgesia. J Pain 2005; 6: 116-24.

11. Fillingim RB, Maixner W. Gender differences in the responses to noxious stimuli. Pain Forum 1995; 4: 209-21.

12. Aubrun F, Salvi N, Coriat P, Riou B. Sex- and age-related differences in morphine requirements for postoperative pain relief. Anesthesiology 2005; 103: 156-60.

13. Cepeda MS, Carr DB. Women experience more pain and require more morphine than men to achieve a similar degree of analgesia. Anesth Analg 2003; 97: 1464-8.

14. Zeidan A, Al-Temyatt S, Mowafi H, Ghattas T. Gender-related difference in postoperative pain after laparoscopic Roux-En-Y gastric bypass in morbidly obese patients. Obes Surg 2013; 23: 18804 .

15. Theodoraki K, Staikou C, Fassoulaki A. Postoperative pain after major bdominal surgery: is it gender related? An observational prospective study. Pain Pract 2014; 14: 613-9.

16. Minto CF, Schnider TW, Egan TD, Youngs E, Lemmens HJ, Gambus PL, et al. Influence of age and gender on the pharmacokinetics and pharmacodynamics of remifentanil. I. Model development. Anesthesiology 1997; 86: 10-23.

17. Ferreira-Valente MA, Pais-Ribeiro JL, Jensen MP. Validity of four pain intensity rating scales. Pain 2011; 152: 2399-404.

18. Jensen MP, Engel JM, McKearnan KA, Hoffman AJ. Validity of pain intensity assessment in persons with cerebral palsy: a comparison of six scales. J Pain 2003; 4: 56-63.

19. Buckenmaier CC 3rd, Galloway KT, Polomano RC, McDuffie M, Kwon N, Gallagher RM. Preliminary validation of the Defense and Veterans Pain Rating Scale (DVPRS) in a military population. Pain Med 2013; 14: 110-23.

20. Fillingim RB, Ness TJ. Sex-related hormonal influences on pain and analgesic responses. Neurosci Biobehav Rev 2000; 24: 485501. 